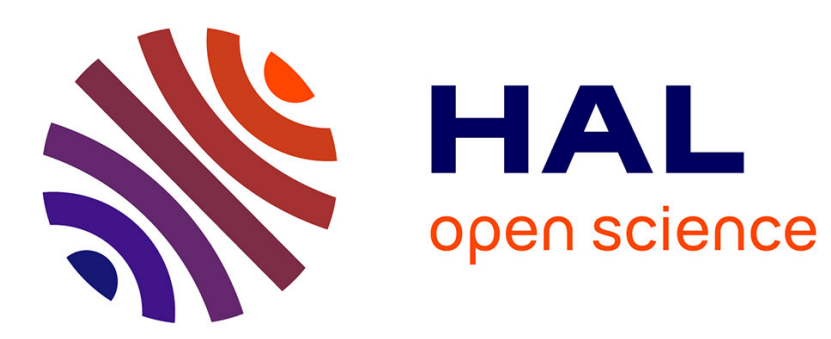

\title{
Variational Formulation and Upper Bounds for Degenerate Scales in Plane Elasticity
}

\author{
Alain Corfdir, Guy Bonnet
}

\section{To cite this version:}

Alain Corfdir, Guy Bonnet. Variational Formulation and Upper Bounds for Degenerate Scales in Plane Elasticity. Journal of Elasticity, 2014, 118 (2), pp.207 - 221. 10.1007/s10659-014-9494-1 . hal-01157344

\section{HAL Id: hal-01157344 \\ https://hal-enpc.archives-ouvertes.fr/hal-01157344}

Submitted on 30 Jun 2015

HAL is a multi-disciplinary open access archive for the deposit and dissemination of scientific research documents, whether they are published or not. The documents may come from teaching and research institutions in France or abroad, or from public or private research centers.
L'archive ouverte pluridisciplinaire $\mathbf{H A L}$, est destinée au dépôt et à la diffusion de documents scientifiques de niveau recherche, publiés ou non, émanant des établissements d'enseignement et de recherche français ou étrangers, des laboratoires publics ou privés. 


\title{
Variational formulation and upper bounds for degenerate scales in plane elasticity
}

\author{
Alain Corfdir, Guy Bonnet
}

Received: date / Accepted: date

\begin{abstract}
Degenerate scales appear when certain plane boundary value problems solved using Boundary Integral Equations do not have a unique solution. The main contribution of this paper is to prove four inequalities that constrain the degenerate scales for plane elasticity. These results are based on a new variational formulation. It is shown that the degenerate scales depend only on Poisson's ratio. The bounds on the degenerate scales for plane elasticity in a given boundary are obtained mainly from the degenerate scales obtained from the Laplace equation for the same boundary, which are well documented.
\end{abstract}

Keywords Plane elasticity · Degenerate scale · Robin constant · Green function

PACS $74 \mathrm{~B} 05 \cdot 74515 \cdot 45 \mathrm{Pxx}$

\section{Introduction}

Degenerate scales appear when solving certain Boundary Value Problems (BVP) related to linear operators and using a formulation of a Boundary Integral Equation (BIE). This formulation introduces single-layer operators, which must be invertible to ensure the uniqueness of the solution in all cases. Unfortunately, the uniqueness of the solution is not always ensured in the case of

\footnotetext{
Alain Corfdir

Université Paris-Est, Laboratoire Navier, UMR 8205, CNRS, ENPC, IFSTTAR, 6 et 8 avenue Blaise Pascal, 77455 Marne-la-Vallée, France

Tel.: +33-1-64153521

Fax: $+33-1-64153562$

E-mail: corfdir@cermes.enpc.fr

Guy Bonnet

Université Paris-Est, Laboratoire Modélisation et Simulation Multi-Echelle, UMR 8208 CNRS, 5 boulevard Descartes, 774454, Marne-la-Vallée, France

E-mail: Guy.Bonnet@univ-mlv.fr
} 
plane problems. The loss of uniqueness occurs when the size of the domain is at a degenerate scale.

The occurrence of the degenerate scale is well known when studying the Laplace equation $[9,20]$. It appears when Dirichlet boundary conditions are applied at the contour of a plane domain. If one considers all the contours $\lambda \Gamma$ that are homothetic to a given contour $\Gamma$, the integral equation for the normal flux $\phi$ at these boundaries reads:

$$
\int_{\lambda \Gamma}-\frac{1}{2 \pi} \ln (\|\mathbf{x}-\mathbf{y}\|) \phi(\mathbf{y}) \mathrm{d} \Gamma_{y}=f(\mathbf{x}),
$$

where $\Gamma$ and $f$ comply with suitable regularity conditions. For given $f$, all these integral equations have only one solution, except for a specific value $\lambda_{d}$ of $\lambda$ for which this equation degenerates and has an infinite number of solutions. The non-dimensional constant $\lambda_{d}=\rho_{0}$ could be called the "degenerate scale factor", but is customarily called the "degenerate scale".

One important consequence is that the proximity of such a degenerate scale can cause the ill conditioning of matrices obtained when using the numerical solution obtained by the Boundary Element Method (BEM) [14]. For a domain having such a scale, the numerical system built by the BEM is singular. In addition, knowing the degenerate scale makes it possible to disregard the scales providing a non-physical supply of energy when using the BEM [2].

It is worth noting that setting up this problem requires:

- the choice of the unit of length (scaling) used to describe the domain and its boundary $\Gamma$,

- the initial boundary $\Gamma$

- the choice of the Green's function (which is defined up to an arbitrary additive constant).

Finding a value of the degenerate scale has a meaning only when all these elements are clearly defined.

There are several ways to obtain the degenerate scale for the Laplace equation, using solutions from complex analytical functions, the BEM and other options. An important point is the relationship between the degenerate scale and certain important parameters of the analytical function theory, i.e. the logarithmic capacity and the Robin constant, whose definitions will be reviewed in section 2 .

Obtaining the degenerate scale is a fully documented process in the case of the Laplace equation, but this question is also relevant in other physical domains. There are scales where boundary integral operators are not invertible, causing loss of uniqueness for the plane problem with elasticity $[10,16,27]$ and the biharmonic equation [12]. If the scaling parameter is small enough, the scaled boundary integral operator is elliptic and then invertible [25]. However, the results for topics other than the Laplace equation are significantly less numerous. Therefore, the objective of this paper is to provide new results on the degenerate scales for plane elasticity and, more specifically, to show that these degenerate scales must comply with precise inequalities related to domains of different forms and to the values of degenerate scales for the Laplace 
equation related to the same domain (which can be obtained from the literature in numerous cases).

In the second section, certain contextual elements related to the Laplace equation and plane elasticity will be reviewed. In particular, the extension of the Robin constant to elasticity problems will be defined using a matrix introduced by Vodička and Mantič [27]. Next, the paper will be devoted exclusively to results for plane elasticity. In section 3 , the variational formulation will be provided for characterizing degenerate scales. In the last sections, this variational formulation will be used to obtain specific inequalities on the degenerate scales for plane elasticity.

2 Context of the degenerate scales for the Laplace equation and for elasticity

This paper contains an extension to plane elasticity of some results obtained for plane problems using the Laplace equation. So, in a first step, some results related to the Laplace equation are reviewed.

2.1 Main results in the case of the Laplace equation: Robin constant and logarithmic capacity

In the case of the Laplace equation, the most commonly used tools for defining the degenerate scale are the logarithmic capacity and the Robin constant related to a given contour.

The Robin constant $V_{\Gamma}$ related to contour $\Gamma$ is defined classically [17] as follows:

$$
\begin{aligned}
& \qquad V_{\Gamma}=\inf \int_{\Gamma} \int_{\Gamma} \ln \left(\frac{1}{\|\mathbf{x}-\mathbf{y}\|}\right) q(\mathbf{x}) q(\mathbf{y}) \mathrm{d} \Gamma_{x} \mathrm{~d} \Gamma_{y}, \\
& \text { with } q \geq 0, \int_{\Gamma} q \mathrm{~d} \Gamma=1 .
\end{aligned}
$$

This produces the logarithmic capacity $C_{\Gamma}$ :

$$
C_{\Gamma}=e^{-V_{\Gamma}}
$$

This definition involves the positiveness of the weighting function $q$, which comes from the nature of $q$ as the density of a positive measure. However, another characterization has been proposed by Hsiao and Kleinman [19]. This alternative characterization of the Robin constant is described in Appendix A. It will be shown thereafter that this second characterization can be extended to plane elasticity for finding the extension of the Robin constant to plane elasticity proposed by Vodička and Mantič [27] .

An important result is that the degenerate scale occurs when $C_{\Gamma}=1$, or $V_{\Gamma}=0$. Consequently, the logarithmic capacity determines the degenerate 
scale $[15,21,29]$. Indeed, considering a contour $\Gamma$ such that $C_{\Gamma} \neq 1$, the degenerate scale is given by: $\rho_{0}=1 / C_{\Gamma}$. The positiveness of the operator is ensured if $C_{\Gamma}<1$. For domains where $C_{\Gamma}>1$, there are certain potentials that lead to negative energy, which is not physically consistent [2].

The logarithmic capacity is known in closed form for numerous special cases, as discussed in the review in [23]. For the cases that are not characterized by a closed form of the logarithmic capacity, this quantity can be computed numerically $[11,13]$. However, upper and lower bounds can also be useful for quickly appraising a range of possible values of the degenerate scale. Therefore, several authors have produced inequalities involving the logarithmic capacity. Some of these results are summarized in Appendix B. These inequalities can be combined with the ones obtained in the following sections to find bounds for degenerate scales in the case of plane elasticity.

2.2 Single layer operator and degenerate scales for plane elasticity

We consider the single layer operator $\mathbf{U}_{\Gamma}$ for plane elasticity in the case of a Lipschitz boundary $\Gamma$

$$
\mathbf{U}_{\Gamma}(\phi)=\int_{\Gamma} U_{i, j}(\mathbf{x}, \mathbf{y}) \phi_{j} \mathrm{~d} \Gamma_{y}
$$

where $U_{i, j}$ are the usual components of the Green tensor for plane elasticity (Kelvin's tensor):

$$
\begin{gathered}
U_{i, j}=\Lambda\left(-\kappa \delta_{i, j} \ln |\mathbf{x}-\mathbf{y}|+\frac{\left(x_{i}-y_{i}\right)\left(x_{j}-y_{j}\right)}{|\mathbf{x}-\mathbf{y}|^{2}}\right), \\
\Lambda=\frac{1}{8 \pi G(1-\nu)} \\
7>\kappa=3-4 \nu>1
\end{gathered}
$$

The degenerate scales come from the single layer integral equation that appears in plane elasticity problems with the displacement known at the boundary. For different homothetic contours $\lambda \Gamma$, we have

$$
\mathbf{U}_{\lambda \Gamma}(\phi)=\mathbf{f}
$$

The main difference from the Laplace equation is that there are now two degenerate scales $\lambda=\rho_{i}, i=1,2$ such that the integral equation has multiple solutions. Obviously, if the integral equation has multiple solutions, then the same equation with $\mathbf{f}=0$ has non-zero solutions. 
2.3 A matrix equivalent to the Robin constant

As shown above, the Robin constant and the logarithmic capacity are useful to define the degenerate scales in the case of the Laplace equation.

In the case of plane elasticity, there are two degenerate scales. It was shown by Vodička and Mantič [27] that these scales can be recovered by defining a specific matrix equivalent to the Robin constant. This matrix is obtained by applying to the isotropic plane elasticity the methods developed by Costabel and Dauge [12] for the biharmonic operator.

Vodička and Mantič have considered the following system where $\boldsymbol{\xi}$ is a given constant vector, $\Gamma$ is a bounded Lipschitz boundary and $(\boldsymbol{\phi}, \boldsymbol{\omega}) \in\left[H^{-1 / 2}(\Gamma)\right]^{2} \times$ $\mathbb{R}^{2}$ are unknown:

$$
\left\{\begin{aligned}
\mathbf{U}_{\Gamma}(\phi)-\boldsymbol{\omega} & =0, \\
\int_{\Gamma} \phi \mathrm{d} \Gamma_{y} & =\boldsymbol{\xi},
\end{aligned}\right.
$$

has a unique solution. In this solution, $\boldsymbol{\omega}$ is a linear function of $\boldsymbol{\xi}$ due to the linearity of the equation. This system is clearly the extension of the problem related to the system of equations $(66,67)$, which is reviewed in appendix A for the Laplace equation.

The linear relation between $\boldsymbol{\omega}$ and $\boldsymbol{\xi}$, which are constant vectors, can be specified by a constant matrix $\mathbf{B}$ such that:

$$
\mathrm{B} \xi=\omega
$$

This definition is similar to the one described in relation (68) in appendix A for the Laplace equation, showing that $\mathbf{B}_{\Gamma}$ is the equivalent for elasticity of the Robin constant.

This matrix is symmetric because the operator $\mathbf{U}_{\Gamma}$ is itself symmetric and invertible if and only if the operator $\mathbf{U}_{\Gamma}$ is invertible $[12,27]$. The operator defined by $\mathbf{B}$ is a second order tensor [28]. The scaling properties and the behavior of $\mathbf{B}$ when modifying the Green function have also been studied in [27]. Due to the previous definitions, it comes that $\Gamma$ is at a degenerate scale iff $\mathbf{B}$ is singular with at least one zero eigenvalue.

Considering the domain with boundary $\Gamma$ and its related $\mathbf{B}_{\Gamma}$, it has been shown in [27] that the matrices $\mathbf{B}_{\lambda \Gamma}$ related to homothetic domains are given by

$$
\mathbf{B}_{\lambda \Gamma}=\mathbf{B}_{\Gamma}-\Lambda \kappa \ln (\lambda \mathbf{I}),
$$

where $\mathbf{I}$ is the unit matrix. A direct consequence is that the degenerate scales related to $\Gamma$ are given by

$$
\rho_{i}=e^{b_{i} / \Lambda \kappa},
$$

where $b_{i}$ are the eigenvalues of $\mathbf{B}_{\Gamma}$.

From an operational point of view, matrix $\mathbf{B}$ can be obtained by using boundary elements [27], even if system ( 9) is not a classical BIE such as 
those used in the formulation of the BEM. However, it does not make it easy to obtain information on degenerate scales for any new domain. Obtaining easily such information is the aim of the following sections.

\section{Pertinent elastic parameters for the degenerate scales and} discriminant matrix

An important aspect related to degenerate scales for elasticity is that the degenerate scales do not depend on all the elastic parameters.

Proposition 1 For a given boundary, the degenerate scales depend only on the Poisson's ratio.

Proof. This property comes directly from the definition of operator U. Indeed, if a distribution of boundary sources ensures that equation (8) has multiple solutions, then it is obvious that the same is true when replacing $\mathbf{U}$ by $K$.U, where $K$ is any constant scalar. Consequently, any degenerate scale for $\mathbf{U}$ is also a degenerate scale for $K$.U. Therefore, the degenerate scale problem does not depend on the prefactor $\Lambda$ in Kelvin's tensor.

In the following, the Green tensor with $\Lambda=1$ will be denoted $\mathbf{U}^{*}$ to distinguish it from the true Kelvin's tensor. If one considers $\mathbf{B}_{\Gamma}^{*}$ built by using Green's tensor $\mathbf{U}^{*}$, one has $\mathbf{B}_{\Gamma}^{*}=\mathbf{B}_{\Gamma} / \Lambda$ with eigenvalues $b_{i}^{*}=b_{i} / \Lambda$ and therefore,

$$
\rho_{i}=e^{b_{i} / \Lambda \kappa}=e^{b_{i}^{*} / \kappa} .
$$

This result shows again that the degenerate scales depend only on the eigenvalues of $\mathbf{B}_{\Gamma}^{*}$ which depend only on the Poisson ratio, as it is the case for $\mathbf{B}_{\Gamma}^{*}$. In the following, only the non-dimensional matrix $\mathbf{B}^{*}$ will be used. This matrix contains all the information leading to the degenerate scales. Therefore, it will be called the "discriminant matrix".

\section{A variational approach to the discriminant matrix}

As explained in the previous section, the discriminant matrix can be obtained by solving a non-classical boundary integral equation. However, as explained in section 2, the Robin constant and the logarithmic capacity are provided by a variational approach. This variational approach is obviously important from a theoretical point of view, and this section is therefore devoted to providing an equivalent variational approach to the discriminant matrix. In addition to its fundamental significance, it will make it possible to provide general results, such as the bounds obtained in the following sections.

Here, we follow an approach used for the Laplace equation in [19] and apply it to the case of elasticity where matrix $\mathbf{B}^{*}$ takes the place of the Robin constant. We assume $\boldsymbol{\Gamma}$ is the bounded Lipschitz boundary of an open connected domain as in [27]. 
We define the following set of functions:

$$
\begin{gathered}
\Phi_{a d}(\boldsymbol{\xi})=\left\{\boldsymbol{\phi}^{\prime} \in\left[H^{-1 / 2}(\Gamma)\right]^{2} / \int_{\Gamma} \boldsymbol{\phi}^{\prime} \mathrm{d} \Gamma_{y}=\boldsymbol{\xi}\right\}, \\
J\left(\boldsymbol{\phi}^{\prime}\right)=\int_{\Gamma} \boldsymbol{\phi}^{\prime} \cdot \mathbf{U}^{*}\left(\boldsymbol{\phi}^{\prime}\right) \mathrm{d} \Gamma_{\mathbf{x}} \\
K(\boldsymbol{\xi})=\inf _{\phi^{\prime} \in \Phi_{a d}(\boldsymbol{\xi})} J\left(\boldsymbol{\phi}^{\prime}\right) .
\end{gathered}
$$

Proposition 2 Under the above assumptions, we have $K(\boldsymbol{\xi})=\boldsymbol{\xi} \mathbf{B}^{*} \boldsymbol{\xi} \quad \forall \boldsymbol{\xi} \in$ $\mathbb{R}^{2}$ and $K(\boldsymbol{\xi})=J(\phi)$ where $\phi, \boldsymbol{\omega}$ are solutions of system $(9)$.

This variational formulation is an extension of the variational principle (69, 70) which is recalled in Appendix A for the Laplace equation and produces the Robin constant.

Proof. We assume that $\phi$ is the solution of system (9) for a given $\boldsymbol{\xi}$. Then, for any $\phi^{\prime} \in \Phi_{a d}(\boldsymbol{\xi})$, we set $\boldsymbol{\eta}=\boldsymbol{\phi}^{\prime}-\boldsymbol{\phi}$, we have $\int_{\Gamma} \boldsymbol{\eta} \mathrm{d} \Gamma=0$ and we can write the following:

$$
\begin{aligned}
J\left(\phi^{\prime}\right) & =J(\phi)+\int_{\Gamma} \boldsymbol{\phi} \cdot \mathbf{U}^{*}(\boldsymbol{\eta}) \mathrm{d} \Gamma_{\mathbf{x}}+\int_{\Gamma} \boldsymbol{\eta} \cdot \mathbf{U}^{*}(\boldsymbol{\phi}) \mathrm{d} \Gamma_{\mathbf{x}}+J(\boldsymbol{\eta})= \\
& =J(\phi)+2 \int_{\Gamma} \boldsymbol{\eta} \cdot \mathbf{U}^{*}(\boldsymbol{\phi}) \mathrm{d} \Gamma_{\mathbf{x}}+J(\boldsymbol{\eta}) .
\end{aligned}
$$

The integral in (18) is null:

$$
\int_{\Gamma} \boldsymbol{\eta} \cdot \mathbf{U}^{*}(\phi) \mathrm{d} \Gamma_{\mathbf{x}}=\int_{\Gamma} \boldsymbol{\eta} \cdot(\boldsymbol{\omega}) \mathrm{d} \Gamma_{\mathbf{x}}=\boldsymbol{\omega} \cdot \int_{\Gamma} \boldsymbol{\eta} \mathrm{d} \Gamma_{\mathbf{x}}=0 .
$$

According to Proposition 1 of [27], we have $J(\boldsymbol{\eta})>0$ for all $\boldsymbol{\eta} \neq 0$ such that $\int_{\Gamma} \boldsymbol{\eta} \mathrm{d} \Gamma_{\mathbf{x}}=0$. We then deduce the following:

$$
J\left(\phi^{\prime}\right)>J(\phi) \text { if } \phi^{\prime} \neq \phi
$$

This result proves that the value of $J(\phi)$ leading to the minimum of $K$ corresponds to the solution of (9). We can finally conclude by the following set of equalities:

$$
K(\boldsymbol{\xi})=J(\phi)=\int_{\Gamma} \phi \cdot \mathbf{U}^{*}(\phi) \mathrm{d} \Gamma_{\mathbf{x}}=\int_{\Gamma} \phi \cdot \omega \mathrm{d} \Gamma_{\mathbf{x}}=\boldsymbol{\xi} \boldsymbol{\omega}=\boldsymbol{\xi} \mathbf{B}^{*} \boldsymbol{\xi}
$$

As $\mathbf{B}^{*}$ is symmetric because $\mathbf{U}^{*}$ is symmetric, $\mathbf{B}^{*}$ is completely defined by using the polarization identity:

$$
\boldsymbol{\xi}_{1} \mathbf{B}^{*} \boldsymbol{\xi}_{2}=\frac{1}{4}\left(K\left(\boldsymbol{\xi}_{1}+\boldsymbol{\xi}_{2}\right)-K\left(\boldsymbol{\xi}_{1}-\boldsymbol{\xi}_{2}\right)\right) .
$$




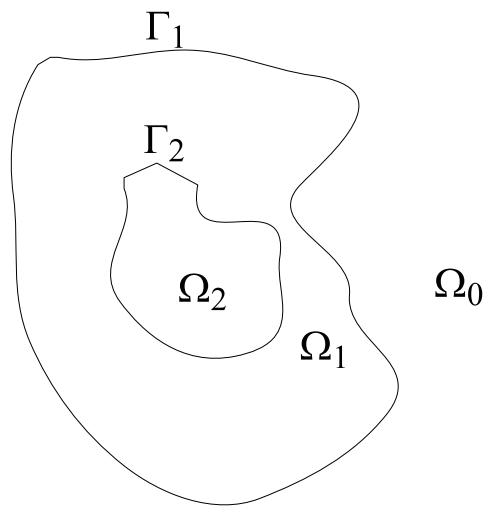

Fig. 1 A simple case of two boundaries $\Gamma_{1}$ and $\Gamma_{2}$

5 A comparison of $\mathrm{B}^{*}$ matrix for two boundaries, one being enclosed in the other

We consider two boundaries, one enclosed in the other (Figure 1). We consider any $\boldsymbol{\xi} \neq 0$ and any $\phi \neq 0$ defined on $\Gamma_{2}$ such that: $\int_{\Gamma_{2}} \phi \mathrm{d} \Gamma=\boldsymbol{\xi}$. We then consider $\phi_{1}$ the solution of

$$
\left\{\begin{aligned}
\mathbf{U}_{\Gamma_{1}}^{*}\left(\phi_{\mathbf{1}}\right)-\boldsymbol{\omega} & =0, \\
\int_{\Gamma_{1}} \phi_{\mathbf{1}} \mathrm{d} \Gamma & =-\boldsymbol{\xi} .
\end{aligned}\right.
$$

We then define $\widetilde{\phi}$ as follows [27]:

$$
\widetilde{\phi}=\left\{\begin{array}{l}
\phi \text { on } \Gamma_{2}, \\
\phi_{1} \text { on } \Gamma_{1} .
\end{array}\right.
$$

As $\int_{\Gamma_{1} \cup \Gamma_{2}} \widetilde{\phi} \mathrm{d} \Gamma=0$, we can write the following [27]:

$$
0<\int_{\Gamma_{1} \cup \Gamma_{2}} \widetilde{\phi} \mathbf{U}_{\Gamma_{1} \cup \Gamma_{2}}^{*}(\widetilde{\phi}) \mathrm{d} \Gamma .
$$

We can split the integral into four parts, as follows:

$$
\begin{aligned}
& \int_{\Gamma_{1} \cup \Gamma_{2}} \widetilde{\phi} \mathbf{U}_{\Gamma_{1} \cup \Gamma_{2}}^{*}(\widetilde{\phi}) \mathrm{d} \Gamma= \\
& \int_{\Gamma_{2}} \phi \mathbf{U}_{\Gamma_{2}}^{*}(\phi) \mathrm{d} \Gamma+\int_{\Gamma_{2}} \phi \mathbf{U}_{\Gamma_{1}}^{*}\left(\phi_{1}\right) \mathrm{d} \Gamma+\int_{\Gamma_{1}} \phi_{1} \mathbf{U}_{\Gamma_{2}}^{*}(\phi) \mathrm{d} \Gamma+\int_{\Gamma_{1}} \phi_{1} \mathbf{U}_{\Gamma_{1}}^{*}\left(\phi_{1}\right) \mathrm{d} \Gamma .
\end{aligned}
$$


Due to the symmetry of the operator $\mathbf{U}^{*}$, the second and the third integral are equal. From the definition of $\phi_{1}$, the following is true:

$$
\int_{\Gamma_{1}} \phi_{1} \mathbf{U}_{\Gamma_{1}}^{*}\left(\phi_{1}\right) \mathrm{d} \Gamma=-\boldsymbol{\xi} \cdot \boldsymbol{\omega} .
$$

As the potential $\phi_{1}$ generates a constant displacement field on $\Gamma_{1}$, the displacement field generated by $\phi_{1}$ is constant (rigid motion) in the domain $\Omega_{1}$ and in particular $\mathbf{U}_{\Gamma_{1}}^{*}\left(\phi_{1}\right)(\mathbf{x})=\boldsymbol{\omega}$ for $\mathbf{x} \in \Gamma_{2}$. Then, we can evaluate the second integral as follows:

$$
\int_{\Gamma_{2}} \phi \mathbf{U}_{\Gamma_{1}}^{*}\left(\phi_{1}\right) \mathrm{d} \Gamma=\boldsymbol{\xi} \cdot \boldsymbol{\omega}
$$

Replacing the last three integrals by their value in the previous inequality leads to:

$$
0<\int_{\Gamma_{2}} \phi \mathbf{U}_{\Gamma_{2}}^{*}(\phi) \mathrm{d} \Gamma+\boldsymbol{\xi} \cdot \boldsymbol{\omega}=\int_{\Gamma_{2}} \phi \mathbf{U}_{\Gamma_{2}}^{*}(\phi) \mathrm{d} \Gamma-K_{1}(\xi)
$$

which gives

$$
K_{1}(\boldsymbol{\xi})<\int_{\Gamma_{2}} \phi \mathbf{U}_{\Gamma_{2}}^{*}(\phi) \mathrm{d} \Gamma .
$$

As the above inequality holds for all $\phi$ such that $\int_{\Gamma_{2}} \phi d \Gamma=\boldsymbol{\xi}$, it is also valid at the minimum of this quantity and therefore

$$
K_{1}(\boldsymbol{\xi})<K_{2}(\boldsymbol{\xi})
$$

It proves the following proposition.

Proposition 3 If $\Gamma_{2}$ is included in $\Gamma_{1}$, then we have the following inequality in the sense of Loewner ordering for symmetric operators [18]:

$$
\mathbf{B}_{1}^{*}<\mathbf{B}_{2}^{*}
$$

This inequality is analogous to inequality (72) for the Robin constant. Let $b_{i, 1} \leq b_{i, 2}$ be the ordered eigenvalues of $\mathbf{B}_{i}^{*}$; then it is easily deduced from the previous proposition that

$$
b_{1,1}<b_{2,1}, b_{1,2}<b_{2,2} \text {. }
$$

From relation (12) it can be seen that the degenerate scales are increasing functions of the eigenvalues of $\mathbf{B}^{*}$, and thus, inequalities similar to (33) are recovered for the degenerate scales.

Knowing the degenerate scale for a circle (see Table 2), it is possible to recover condition (79) and then condition (80) by applying Jung's theorem on the smallest circle enclosing a set. 
6 Comparison of $\mathrm{B}^{*}$ for different values of $\kappa$

As seen above, parameter $\Lambda$ does not affect the values of degenerate scales, in contrast to parameter $\kappa$. This section is therefore devoted mainly to studying the effect of the parameter $\kappa$ on the matrix $\mathbf{B}^{*}$. The result is contained in the following proposition:

Proposition 4 If $\kappa \geq \kappa^{\prime}$, we have the following inequality in the sense of symmetric operators:

$$
\mathbf{B}_{\kappa}^{*}-\kappa V_{\Gamma} \mathbf{I} \geq \mathbf{B}_{\kappa^{\prime}}^{*}-\kappa^{\prime} V_{\Gamma} \mathbf{I}
$$

where $\mathbf{I}$ is the second order unit tensor; $V_{\Gamma}$ is the Robin constant; and $\mathbf{B}_{\kappa}^{*}$ is the matrix $\mathbf{B}^{*}$ computed using $\kappa$.

Proof. We consider $\boldsymbol{\xi} . \mathbf{B}_{\kappa}^{*} \boldsymbol{\xi}$. We write the following matrices:

$$
\begin{gathered}
\mathbf{A}=\left(\begin{array}{cc}
\ln (|\mathbf{x}-\mathbf{y}|) & 0 \\
0 & \ln (|\mathbf{x}-\mathbf{y}|)
\end{array}\right), \\
\mathbf{M}=\left(\begin{array}{cc}
\frac{\left(x_{1}-y_{1}\right)^{2}}{|\mathbf{x}-\mathbf{y}|^{2}} & \frac{\left(x_{1}-y_{1}\right)\left(x_{2}-y_{2}\right)}{|\mathbf{x}-\mathbf{y}|^{2}} \\
\frac{\left(x_{1}-y_{1}\right)\left(x_{2}-y_{2}\right)}{|\mathbf{x}-\mathbf{y}|^{2}} & \frac{\left(x_{2}-y_{2}\right)^{2}}{|\mathbf{x}-\mathbf{y}|^{2}}
\end{array}\right) .
\end{gathered}
$$

These matrices are parts of the Green tensor $\mathbf{U}^{*}$, which is readily obtained by $\mathbf{U}^{*}=-\kappa \mathbf{A}+\mathbf{M}$

From the variational formulation and current inequalities, one obtains the following:

$$
\begin{aligned}
\boldsymbol{\xi} . \mathbf{B}_{\kappa}^{*} \cdot \boldsymbol{\xi}= & \inf _{\int_{\Gamma} \Phi=\boldsymbol{\xi}} \int_{\Gamma} \int_{\Gamma}-\kappa \boldsymbol{\phi} \mathbf{A} \boldsymbol{\phi}+\boldsymbol{\phi} \mathbf{M} \boldsymbol{\phi} \mathrm{d} \Gamma_{\mathbf{x}} \mathrm{d} \Gamma_{\mathbf{y}} \\
\geq & \inf _{\int_{\Gamma} \phi=\boldsymbol{\xi}} \int_{\Gamma} \int_{\Gamma}-\kappa^{\prime} \boldsymbol{\phi} \mathbf{A} \boldsymbol{\phi}+\boldsymbol{\phi} \mathbf{M} \boldsymbol{\phi} \mathrm{d} \Gamma_{\mathbf{x}} \mathrm{d} \Gamma_{\mathbf{y}} \\
& +\inf _{\int_{\Gamma} \Phi=\boldsymbol{\xi}} \int_{\Gamma} \int_{\Gamma}-\left(\kappa-\kappa^{\prime}\right) \boldsymbol{\phi} \mathbf{A} \boldsymbol{\phi} \mathrm{d} \Gamma_{\mathbf{x}} \mathrm{d} \Gamma_{\mathbf{y}} .
\end{aligned}
$$

As $\kappa-\kappa^{\prime} \geq 0$, we have

$$
\inf _{\int_{\Gamma} \phi=\boldsymbol{\xi}} \int_{\Gamma} \int_{\Gamma}-\left(\kappa-\kappa^{\prime}\right) \boldsymbol{\phi} \mathbf{A} \boldsymbol{\phi} \mathrm{d} \Gamma_{\mathbf{x}} \mathrm{d} \Gamma_{\mathbf{y}}=\left(\kappa-\kappa^{\prime}\right) \inf _{\int_{\Gamma} \phi=\boldsymbol{\xi}} \int_{\Gamma} \int_{\Gamma}-\boldsymbol{\phi} \mathbf{A} \boldsymbol{\phi} \mathrm{d} \Gamma_{\mathbf{x}} \mathrm{d} \Gamma_{\mathbf{y}}
$$

and

$$
\inf _{\int_{\Gamma} \phi=\boldsymbol{\xi}} \int_{\Gamma} \int_{\Gamma}-\phi \mathbf{A} \phi \mathrm{d} \Gamma_{\mathbf{x}} \mathrm{d} \Gamma_{\mathbf{y}}=V_{\Gamma} \boldsymbol{\xi} . \mathbf{I} . \boldsymbol{\xi}
$$

where $V_{\Gamma}$ is the Robin constant, and

$$
\inf _{\int_{\Gamma} \phi=\boldsymbol{\xi}} \int_{\Gamma} \int_{\Gamma}-\kappa^{\prime} \boldsymbol{\phi} \mathbf{A} \phi+\phi \mathbf{M} \boldsymbol{\phi} \mathrm{d} \Gamma_{\mathbf{x}} \mathrm{d} \Gamma_{\mathbf{y}}=\boldsymbol{\xi} . \mathbf{B}_{\kappa^{\prime}}^{*} \cdot \boldsymbol{\xi} .
$$

From the above equations $(37,38,39,40)$ we conclude that: 


$$
\forall \boldsymbol{\xi} \quad \boldsymbol{\xi} . \mathbf{B}_{\kappa}^{*} \cdot \boldsymbol{\xi} \geq \boldsymbol{\xi} . \mathbf{B}_{\kappa^{\prime}}^{*} \boldsymbol{\xi}-\left(\kappa-\kappa^{\prime}\right) V_{\Gamma} \boldsymbol{\xi} \text {.I. } \boldsymbol{\xi} .
$$

That ends the proof of the proposition.

For the critical scales, we deduce

$$
\left(\frac{\rho_{1}}{\rho_{0}}\right)^{\kappa} \geq\left(\frac{\rho_{1}^{\prime}}{\rho_{0}}\right)^{\kappa^{\prime}},\left(\frac{\rho_{2}}{\rho_{0}}\right)^{\kappa} \geq\left(\frac{\rho_{2}^{\prime}}{\rho_{0}}\right)^{\kappa^{\prime}}
$$

where $\rho_{0}$ is the degenerate scale for the Laplace equation related to $\Gamma$. The above inequalities become equalities (which means that $\left(\rho_{i} / \rho_{0}\right)^{\kappa}$ does not depend on $\kappa$ ) in the case of an ellipse or a segment.

\section{Inequality on the trace of $B^{*}$}

We intend now to prove the following inequality on the trace of $\mathbf{B}^{*}$ :

Proposition 5 The following inequality holds:

$$
\operatorname{tr}^{*} \leq \kappa\left(2 V_{\Gamma}+1 / \kappa\right)
$$

where $V_{\Gamma}$ is the Robin constant.

Proof. We consider: $\boldsymbol{\xi}_{1}=\left(\begin{array}{l}1 \\ 0\end{array}\right), \boldsymbol{\xi}_{2}=\left(\begin{array}{l}0 \\ 1\end{array}\right)$. We can write the following:

$$
\operatorname{tr}\left(\mathbf{B}^{*}\right)=\boldsymbol{\xi}_{1} \cdot \mathbf{B}^{*} \cdot \boldsymbol{\xi}_{1}+\boldsymbol{\xi}_{2} \cdot \mathbf{B}^{*} \cdot \boldsymbol{\xi}_{2}
$$

We denote $\varphi \in H^{-1 / 2}(\Gamma)$, analogous to $\phi$ for the Laplace equation [19], i.e. the value of $\varphi^{\prime}$ producing the Robin constant, as follows:

$$
\begin{aligned}
V_{\Gamma} & =-\int_{\Gamma} \int_{\Gamma} \ln |\mathbf{x}-\mathbf{y}| \varphi(\mathbf{x}) \varphi(\mathbf{y}) \mathrm{d} \Gamma_{\mathbf{x}} \mathrm{d} \Gamma_{\mathbf{y}}= \\
& =\inf _{\int \varphi^{\prime} \mathrm{d} \Gamma=1}-\int_{\Gamma} \int_{\Gamma} \ln |\mathbf{x}-\mathbf{y}| \varphi^{\prime}(\mathbf{x}) \varphi^{\prime}(\mathbf{y}) \mathrm{d} \Gamma_{\mathbf{x}} \mathrm{d} \Gamma_{\mathbf{y}} .
\end{aligned}
$$

We write $\operatorname{tr}\left(\mathbf{B}^{*}\right)$ using the full expression of $\mathbf{U}^{*}$, as follows:

$$
\begin{aligned}
& \boldsymbol{\xi}_{1} \cdot \mathbf{B}^{*} \cdot \boldsymbol{\xi}_{1} \leq \int_{\Gamma}\left(\begin{array}{c}
\varphi \\
0
\end{array}\right)^{T} \mathbf{U}_{\Gamma}^{*}\left(\begin{array}{c}
\varphi \\
0
\end{array}\right) \mathrm{d} \Gamma= \\
= & \kappa V_{\Gamma}+\int_{\Gamma} \int_{\Gamma}\left(\begin{array}{c}
\varphi(\mathbf{x}) \\
0
\end{array}\right)^{T}\left(\begin{array}{cc}
\frac{\left(x_{1}-y_{1}\right)^{2}}{r^{2}} & \frac{\left(x_{1}-y_{1}\right)\left(x_{2}-y_{2}\right)}{r^{2}} \\
\frac{\left(x_{1}-y_{1}\right)\left(x_{2}-y_{2}\right)}{r^{2}} & \frac{\left(x_{2}-y_{2}\right)^{2}}{r^{2}}
\end{array}\right)\left(\begin{array}{c}
\varphi(\mathbf{y}) \\
0
\end{array}\right) \mathrm{d} \Gamma_{\mathbf{y}} \mathrm{d} \Gamma_{\mathbf{y}} \\
= & \kappa V_{\Gamma}+\int_{\Gamma} \int_{\Gamma} \varphi(\mathbf{x}) \frac{\left(x_{1}-y_{1}\right)^{2}}{r^{2}} \varphi(\mathbf{y}) \mathrm{d} \Gamma_{\mathbf{y}} \mathrm{d} \Gamma_{\mathbf{y}} .
\end{aligned}
$$


In the same way, we have

$$
\boldsymbol{\xi}_{2} \cdot \mathbf{B}^{*} \cdot \boldsymbol{\xi}_{2} \leq \kappa V_{\Gamma}+\int_{\Gamma} \int_{\Gamma} \varphi(\mathbf{x}) \frac{\left(x_{2}-y_{2}\right)^{2}}{r^{2}} \varphi(\mathbf{y}) \mathrm{d} \Gamma_{\mathbf{y}} \mathrm{d} \Gamma_{\mathbf{y}} .
$$

Adding the two inequalities, we obtain

$$
\begin{aligned}
\operatorname{tr} \mathbf{B}^{*} & =\boldsymbol{\xi}_{1} \cdot \mathbf{B}^{*} \cdot \boldsymbol{\xi}_{1}+\boldsymbol{\xi}_{2} \cdot \mathbf{B}^{*} \cdot \boldsymbol{\xi}_{2} \\
& \leq 2 \kappa V_{\Gamma}+\int_{\Gamma} \int_{\Gamma} \varphi(\mathbf{x}) \frac{\left(x_{1}-y_{1}\right)^{2}+\left(x_{2}-y_{2}\right)^{2}}{r^{2}} \varphi(\mathbf{y}) \mathrm{d} \Gamma_{\mathbf{y}} \mathrm{d} \Gamma_{\mathbf{y}} .
\end{aligned}
$$

The last integral in the above equation can be evaluated as

$$
\begin{aligned}
& \int_{\Gamma} \int_{\Gamma} \varphi(\mathbf{x}) \frac{\left(x_{1}-y_{1}\right)^{2}+\left(x_{2}-y_{2}\right)^{2}}{r^{2}} \varphi(\mathbf{y}) \mathrm{d} \Gamma_{\mathbf{y}} \mathrm{d} \Gamma_{\mathbf{y}} \\
& =\int_{\Gamma} \int_{\Gamma} \varphi(\mathbf{x}) \varphi(\mathbf{y}) \mathrm{d} \Gamma_{\mathbf{y}} \mathrm{d} \Gamma_{\mathbf{y}}=\int_{\Gamma} \varphi(\mathbf{x}) \mathrm{d} \Gamma_{\mathbf{x}} \int_{\Gamma} \varphi\left(\mathbf{y} \mathrm{d} \Gamma_{\mathbf{y}}=1 .\right.
\end{aligned}
$$

Then, we can conclude that $\operatorname{tr}^{*} \leq \kappa\left(2 V_{\Gamma}+1 / \kappa\right)$.

This inequality can be translated in terms of degenerate scales. We denote by $b_{1} \leq b_{2}$ the two eigenvalues of $\mathbf{B}^{*}$. The two critical scales are $\rho_{1}=e^{b_{1} / \kappa}$ and $\rho_{2}=e^{b_{2} / \kappa}$. We denote by $\rho_{0}$ the critical scale for the Laplace problem and we can write:

$$
\rho_{1} \rho_{2}=e^{\operatorname{tr} \mathbf{B}^{*} / \kappa} \leq e^{2 V_{\Gamma}+1 / \kappa}=\rho_{0}^{2} e^{1 / \kappa} .
$$

Considering Table 2, we note that we have the equality in the case of an ellipse or a segment.

\section{Upper bounds for the eigenvalues of $B^{*}$ and for the degenerate scales}

Proposition 6 If the boundary $\Gamma$ has a rotation symmetry with an angle different from $k \pi, k$ being an integer $\geqslant 3$, then we have the following inequality for the eigenvalues:

$$
b_{1}=b_{2} \leq \kappa\left(V_{\Gamma}+1 / 2 \kappa\right) .
$$

Proof. The equality $b_{1}=b_{2}$ is proved in [28]. Then, the proposition is a direct consequence of the previous one obtained for the trace. In terms of degenerate scales, we can write:

$$
\rho_{1}=\rho_{2} \leq \rho_{0} e^{1 / 2 \kappa} .
$$

It must be noted that the inequality becomes an equality in the case of a circle (Table 2).

We turn now to the general case.

Proposition 7 We have the following inequality for the largest eigenvalue of $\mathbf{B}^{*}$

$$
b_{1} \leq \kappa\left(V_{\Gamma}+1 / \kappa\right)
$$


Proof. We choose $\boldsymbol{\xi}$ an eigenvector corresponding to the largest eigenvalue of $\mathbf{B}^{*}$. We can assume that $\|\boldsymbol{\xi}\|=1$. We can choose the axes so that the first vector of the basis is $\boldsymbol{\xi}$. Then, if $\varphi$ is the solution of problem (45), we have

$$
\phi_{\boldsymbol{\xi}}=\left(\begin{array}{l}
\varphi \\
0
\end{array}\right) \in \mathrm{U}_{a d}(\boldsymbol{\xi})
$$

and then

$$
b_{1}=\boldsymbol{\xi} \cdot \mathbf{B}^{*} \cdot \boldsymbol{\xi} \leq \int_{\Gamma} \phi_{\boldsymbol{\xi}} \mathrm{U}_{\Gamma}^{*}\left(\phi_{\boldsymbol{\xi}}\right) \mathrm{d} \Gamma .
$$

Considering that $\varphi \geq 0$ as a classical result of potential theory, we deduce the following:

$$
\begin{aligned}
b_{1} & \leq \int_{\Gamma} \phi_{\boldsymbol{\xi}} \mathrm{U}_{\Gamma}^{*}\left(\phi_{\boldsymbol{\xi}}\right) \mathrm{d} \Gamma \\
& =\kappa V_{\Gamma}+\int_{\Gamma} \int_{\Gamma} \varphi(\mathbf{x}) \frac{\left(x_{1}-y_{1}\right)^{2}}{r^{2}} \varphi(\mathbf{y}) \mathrm{d} \Gamma_{\mathbf{x}} \mathrm{d} \Gamma_{\mathbf{y}} \\
& \leq \kappa V_{\Gamma}+\int_{\Gamma} \int_{\Gamma} \varphi\left((\mathbf{x}) \varphi(\mathbf{y}) \mathrm{d} \Gamma_{\mathbf{x}} \mathrm{d} \Gamma_{\mathbf{y}}\right. \\
& \leq \kappa V_{\Gamma}+1 .
\end{aligned}
$$

For the largest degenerate scale, we deduce that

$$
\rho_{1} \leq \rho_{0} e^{1 / \kappa}
$$

This inequality becomes an equality in the case of a segment.

\section{Conclusion}

Table 1 shows the main results.

The characterization of $\mathbf{B}^{*}$ as the result of a minimizing process appears to be very useful. It happens to be appropriate to consider the ratio $\rho_{1} / \rho_{0}$ for which certain constraining inequalities have been proved using the variational formulation of $\mathbf{B}^{*}$. These inequalities are sharp in the sense that they become equalities in certain particular cases. These results highlight the link between the problems of the degenerate scales for plane elasticity and for plane Laplace problems. The upper bounds that have been found for degenerate scales make it possible to check easily whether the domain is larger than its critical scale. Finally, the examination of Table 2 suggests the following conjecture: the degenerate scales for plane elasticity are always greater than the degenerate scale for the Laplace equation. This conjecture will be studied in a future work. 
Table 1 Summary of results

\begin{tabular}{|c|c|c|}
\hline $\begin{array}{l}\text { Case of two domains } \\
\Omega^{\prime} \varsubsetneqq \Omega \text { with the same } \\
\kappa\end{array}$ & $\begin{array}{l}\mathbf{B}^{* \prime}>\mathbf{B}^{*} \\
\rho_{1}^{\prime}>\rho_{1} ; \rho_{2}^{\prime}>\rho_{2}\end{array}$ & \\
\hline $\begin{array}{l}\text { Case of the same do- } \\
\text { main with two values of } \\
\kappa, \kappa \geq \kappa^{\prime}\end{array}$ & $\begin{array}{l}\mathbf{B}_{\kappa}^{*}-\kappa V_{\Gamma} \mathbf{I} \geq \mathbf{B}_{\kappa^{\prime}}^{*}-\kappa^{\prime} V_{\Gamma} \mathbf{I} \\
\left(\frac{\rho_{1}}{\rho_{0}}\right)^{\kappa} \geq\left(\frac{\rho_{1}^{\prime}}{\rho_{0}}\right)^{\kappa^{\prime}} \\
\left(\frac{\rho_{2}}{\rho_{0}}\right)^{\kappa} \geq\left(\frac{\rho_{2}^{\prime}}{\rho_{0}}\right)^{\kappa^{\prime}}\end{array}$ & $\begin{array}{l}\text { This inequality be- } \\
\text { comes an equality for } \\
\text { an ellipse or a segment. }\end{array}$ \\
\hline $\begin{array}{l}\text { Upper bound of } \\
\text { the trace of } \mathbf{B}^{*}\end{array}$ & $\begin{array}{l}\kappa\left(2 V_{\Gamma}+1 / \kappa\right) \geq \operatorname{tr} \mathbf{B}^{*} \\
\rho_{0}^{2} e^{1 / \kappa} \geq \rho_{1} \rho_{2}\end{array}$ & $\begin{array}{l}\text { This inequality be- } \\
\text { comes an equality in } \\
\text { the case of an ellipse } \\
\text { or a segment. }\end{array}$ \\
\hline $\begin{array}{l}\text { Upper bound of the de- } \\
\text { generate scale: general } \\
\text { case }\end{array}$ & $\rho_{0} e^{1 / \kappa} \geq \rho_{1} \geq \rho_{2}$ & $\begin{array}{l}\text { This inequality be- } \\
\text { comes an equality for } \\
\text { the largest degenerate } \\
\text { scale of a segment. }\end{array}$ \\
\hline $\begin{array}{l}\text { Upper bound of the } \\
\text { degenerate scale: case } \\
\text { with an axial symme- } \\
\text { try of angle } 2 \pi / k k \geq 3\end{array}$ & $\rho_{0} e^{1 / 2 \kappa} \geq \rho_{1}=\rho_{2}$ & $\begin{array}{l}\text { This inequality be- } \\
\text { comes an equality for } \\
\text { a circle. }\end{array}$ \\
\hline
\end{tabular}

A An alternative to the definition of the logarithmic capacity and of the Robin constant

Hsiao and Kleinman [19] have considered the following problem:

$$
\int_{\Gamma}-\frac{1}{2 \pi} \ln (\|\mathbf{x}-\mathbf{y}\|) \phi(\mathbf{y}) \mathrm{d} \Gamma_{y}=\omega,
$$

with

$$
\int_{\Gamma} \phi(\mathbf{y}) \mathrm{d} \Gamma_{y}=\xi
$$

where $\xi$ is a given constant and $\phi$ and $\omega$ are unknown. This constant $\xi$ is equal to 1 in the original paper [19] but has been extended above to further illuminate the analogy with the similar problem for elasticity.

It has been shown that the system $(66,67)$ has a unique solution $(\phi, \omega)$ with an obvious relation coming from the linearity of the problem

$$
\xi V_{\Gamma}=\omega
$$

and $V_{\Gamma}$ is the previously defined Robin constant. This (non-classical) formulation of the Robin constant is in fact exactly analogous to the formulation of the $\mathbf{B}$ matrix in elasticity, which is reviewed in the subsection 2.3. Having considered the variational formulation related to this problem, it has been proved [19] that $V_{\Gamma}$ is also given (for $\xi=1$ ) by

$$
V_{\Gamma}=\inf _{\phi \in U_{a d}} \int_{\Gamma} \int_{\Gamma} \ln \left(\frac{1}{\|\mathbf{x}-\mathbf{y}\|}\right) \phi(\mathbf{x}) \phi(\mathbf{y}) \mathrm{d} \Gamma_{x} \mathrm{~d} \Gamma_{y}
$$

where

$$
U_{a d}=\left\{\phi \in H^{-1 / 2}(\Gamma), \int_{\Gamma} \phi(\mathbf{y}) \mathrm{d} \Gamma=1\right\} .
$$

This formulation means that the inf can now be sought without restricting the functions $\phi$ to be positive. This variational principle is extended to plane elasticity in section 4 . 


\section{B Inequalities for the logarithmic capacity}

The main results obtained in potential theory that constrain the logarithmic capacity (and obviously the Robin constant) are as follows:

a/ Capacity of growing domains

If $\Omega_{2} \subset \Omega_{1}$, then we have:

$$
C_{\partial \Omega_{2}} \leqslant C_{\partial \Omega_{1}}
$$

This result shows that the logarithmic capacity is a function that increases for growing domains. However, considering the relation between the Robin constant and the logarithmic capacity, the "Robin constant" decreases for growing domains as shown below:

$$
V_{\partial \Omega_{1}} \leqslant V_{\partial \Omega_{2}}
$$

b/ Capacity of a domain contained within a circle

If $\partial \Omega$ is included in a circle of diameter $d_{\partial \Omega}$, we have

$$
C_{\partial \Omega} \leqslant \frac{d_{\partial \Omega}}{2} .
$$

c/ Capacity of a domain with a finite projection on a straight line

If $p$, the maximum length of the projection of $\partial \Omega$ on a straight line, is known, then [22] we have the following:

$$
C_{\partial \Omega} \geqslant \frac{p}{4}
$$

d/ Relationship between capacity and area of domain inside $\partial \Omega$

If one introduces the area $A_{\partial \Omega}$, one can write [22]

$$
C_{\partial \Omega} \geqslant\left(\frac{A_{\partial \Omega}}{\pi}\right)^{\frac{1}{2}}
$$

More recently, a sharper inequality was given in [1] using $\Psi$, a special increasing function defined in this paper, with the form

$$
C_{\partial \Omega} \geqslant \frac{1}{2} d_{\partial \Omega} \Psi\left(\frac{A_{\partial \Omega}}{\frac{1}{4 \pi} d_{\partial \Omega}^{2}}\right)
$$

e/ Capacity of a polygon

For a polygon $D_{n}$ with $\mathrm{n}$ sides and area $A_{D_{n}}$ [24] we have the following inequality:

$$
\frac{C_{D_{n}}^{2}}{A_{D_{n}}} \geqslant \frac{n \tan (\pi / n) \Gamma^{2}(1+1 / n)}{\pi 2^{(4 / n)} \Gamma^{2}(1 / 2+1 / n)} .
$$

This inequality becomes an equality for regular polygons. For $n=3,4$ the above inequality was already given by Pólia and Szegö [22].

\section{Results of previous works on degenerate scales in plane elasticity}

There are far fewer results for the case of plane elasticity than for the case of the Laplace equation. Closed form solutions are known only for a few cases of contours. Table 2 presents the related results from a few references. Concerning the results in $[3,7]$, it is worth noting that these papers present the results for a complex potential. This complex potential leads to the following Green tensor, which is different from $(5)[6,8]$ :

$$
U_{i, j}=\Lambda\left(-\kappa \delta_{i, j} \ln |\mathbf{x}-\mathbf{y}|-\frac{1}{2} \delta_{i, j}+\frac{\left(x_{i}-y_{i}\right)\left(x_{j}-y_{j}\right)}{|\mathbf{x}-\mathbf{y}|^{2}}\right) .
$$


Table 2 Degenerate scales for Laplace and elasticity problems for different boundaries

\begin{tabular}{|c|c|c|}
\hline Boundary & $\begin{array}{l}\text { Critical scale } \\
\text { for Laplace } \\
\text { equation }\end{array}$ & $\begin{array}{l}\text { Critical scales for elasticity (stan- } \\
\text { dard fundamental solutions) }\end{array}$ \\
\hline Circle of radius 1 & $\rho_{0}=1$ & $\rho_{1}=\rho_{2}=e^{1 / 2 \kappa}$ \\
\hline $\begin{array}{l}\text { Ellipse with half axis a and } \mathrm{b} \\
\text { such that } a+b=2\end{array}$ & $\rho_{0}=1$ & $\begin{array}{l}\rho_{1}=e^{(1-m) / 2 \kappa} ; \rho_{2}=e^{(1+m) / 2 \kappa} \\
\text { with } m=(a-b) /(a+b)\end{array}$ \\
\hline Segment of length 4 & $\rho_{0}=1$ & $\rho_{1}=1 ; \rho_{2}=e^{1 / \kappa}[27]$ \\
\hline $\begin{array}{l}\text { Hypotrochoid, approximat- } \\
\text { ing an equilateral triangle, } \\
\text { image of the unit circle by } \\
z=\left(\zeta+1 / 3 \zeta^{2}\right)\end{array}$ & $\rho_{0}=1$ & $\rho_{1}=\rho_{2}=e^{-1 / 18 \kappa^{2}+1 / 2 \kappa}$ \\
\hline $\begin{array}{l}\text { Hypotrochoid, approximat- } \\
\text { ing a square, image of the } \\
\text { unit circle by } z=\left(\zeta-1 / 6 \zeta^{3}\right)\end{array}$ & $\rho_{0}=1$ & $\rho_{1}=\rho_{2}=e^{-1 / 36 \kappa^{2}+1 / 2 \kappa}$ \\
\hline $\begin{array}{l}\text { Ellipse-like contour, } \\
\text { image of } \\
\text { the unit circle by } \\
z=(\zeta-m /(4 \zeta-1)) \\
\text { with } 0<m \leq 1\end{array}$ & $\rho_{0}=1$ & $\begin{array}{l}\rho_{1}=e^{\frac{s_{1}}{32 \kappa}\left(-\frac{s_{2}}{\kappa-s_{2}}-1\right)+\frac{1}{2 \kappa}} \\
\rho_{2}=e^{\frac{s_{1}}{32 \kappa}\left(-\frac{s_{2}}{\kappa-s_{2}}+1\right)+\frac{1}{2 \kappa}} \\
\text { with } s_{1}=-900 m /(225-4 m) \\
s_{2}=-4 m /(225-4 m)[5]\end{array}$ \\
\hline
\end{tabular}

These results from [3,7] have been processed to correspond with the Green tensor defined previously in (5), corresponding to the factor $\mathrm{e}^{1 / 2 \kappa}$ in the last column of Table 2.

Among other notable results, an asymptotic property of degenerate scale for the case of multiple holes has recently been given [26], the case of multiple rigid lines has been investigated [4], and two forms of sufficient inequality conditions were obtained for the definite positiveness of the operator (using the usual Green function ) [27,28], as follows:

$$
\begin{aligned}
d_{\partial \Omega} & <2 e^{\frac{1}{2 \kappa}} . \\
l_{\max } & <\sqrt{3} e^{\frac{1}{2 \kappa}} .
\end{aligned}
$$

where $d_{\partial \Omega}$ is the minimal diameter of a circle that contains $\Gamma$, and $l_{\max }$ is equal to

$$
l_{\max }=\max _{\mathbf{x}, \mathbf{y} \in \Gamma}\|\mathbf{x}-\mathbf{y}\| .
$$

\section{References}

1. Barnard, R.W., Pearce, K., Solynin, A.Y.: An isoperimetric inequality for logarithmic capacity. Annales Academiae Scientiarum Fennicae, Mathematica 27, 419-436 (2002)

2. Bonnet, G., Corfdir, A., Nguyen, M.T.: On the solution of exterior plane problems by the boundary element method: a physical point of view. Engineering Analysis with Boundary Elements 38, 40-48 (2014)

3. Chen, J.T., Kuo, S.R., Lin, J.H.: Analytical study and numerical experiments for degenerate scale problems in the boundary element method for two-dimensional elasticity. International Journal for Numerical Methods in Engineering 54(12), 1669-1681 (2002)

4. Chen, Y.Z.: Numerical solution for degenerate scale problem arising from multiple rigid lines in plane elasticity. Applied Mathematics and Computation 218, 96-106 (2011)

5. Chen, Y.Z., Lin, X.Y., Wang, Z.X.: Evaluation of the degenerate scale for BIE in plane elasticity by using conformal mapping. Engineering Analysis with Boundary Elements 33, $147-158(2009)$ 
6. Chen, Y.Z., Lin, X.Y., Wang, Z.X.: Influence of different integral kernels on the solutions of boundary integral equations in plane elasticity. Journal of Mechanics of Materials and Structures 5(4), 679-692 (2010)

7. Chen, Y.Z., Wang, Z.X., Lin, X.Y.: Eigenvalue and eigenfunction analysis arising from degenerate scale problem of BIE in plane elasticity. Engineering Analysis with Boundary Elements 31, 994-1002 (2007)

8. Chen, Y.Z., Wang, Z.X., Lin, X.Y.: A new kernel in BIE and the exterior boundary value problem in plane elasticity. Acta Mechanica 206, 207-224 (2009)

9. Christiansen, S.: Integral equations without a unique solution can be made useful for solving some plane harmonic problems. Journal of the Institute of Mathematics and Its Applications 16, 143-159 (1975)

10. Constanda, C.: On non-unique solutions of weakly singular integral equations in plane elasticity. Quarterly Journal of Mechanics and Applied Mathematics 47(2), 261-268 (1994)

11. Corfdir, A., Bonnet, G.: Degenerate scale for the Laplace problem in the half-plane; approximate logarithmic capacity for two distant boundaries. Engineering Analysis with Boundary Elements 37, 836-841 (2013)

12. Costabel, M., Dauge, M.: Invertibility of the biharmonic single layer potential operator. Integral Equations and Operator Theory 24(1), 46-67 (1996)

13. Dijkstra, W., Hochstenbach, M.: Numerical approximation of the logarithmic capacity. http://www.win.tue.nl/ hochsten/pdf/logcap.pdf pp. 1-19 (2009)

14. Dijkstra, W., Mattheij, R.M.M.: A relation between the logarithmic capacity and the condition number of the BEM matrices. Communications in Numerical Methods in Engineering 23, 665-680 (2007)

15. Hayes, J., Kellner, R.: The eigenvalue problem for a pair of coupled integral equations arising in the numerical solution of Laplace's equation. SIAM Journal on Applied Mathematics 22(3), 503-513 (1972)

16. Heise, U.: The spectra of some integral operators for plane elastostatical boundary value problems. Journal of Elasticity 8(1), 47-79 (1978)

17. Hille, E.: Analytic function theory. Vol. II, first edn. Ginn, Boston (1962)

18. Horn, R.A., Johnson, C.R.: Matrix analysis, second edn. Cambridge University Press, Cambridge (2013)

19. Hsiao, G.C., Kleinmann, R.E.: On a uniform characterization of capacity. In: J. Král (ed.) Potential Theory, pp. 103-126. Plenum Press, Prague, Czechoslovakia (1987)

20. Jaswon, M.A.: Integral equation methods in potential theory, I. Proceedings of the Royal Society of London A275, 23-32 (1963)

21. Kuo, S.R., Chen, J.T., Kao, S.K.: Linkage between the unit logarithmic capacity in the theory of complex variables and the degenerate scale in the BEM/BIEMs. Applied Mathematical Letters 26(9), 929-938 (2013)

22. Pólia, G., Szegö, G.: Isoperimetric inequalities in mathematical physics. Princeton University, Princeton (1951)

23. Rumely, R.S.: Capacity Theory on Algebraic Curves. Springer-Verlag, Berlin (1989)

24. Solynin, A.Y., Zalgaller, V.A.: An isoperimetric inequality for logarithmic capacity of polygons. Annals of Mathematics 159, 277-303 (2004)

25. Steinbach, O.: A note on the ellipticity of the single layer potential in two-dimensional linear elastostatics. J. Math. Anal. Appl. 294, 1-6 (2004)

26. Vodička, R.: An asymptotic property of degenerate scales for multiple holes in plane elasticity. Applied Mathematics and Computation 220, 166-175 (2013)

27. Vodička, R., Mantič, V.: On invertibility of elastic single-layer potential operator. Journal of Elasticity 74(2), 147-173 (2004)

28. Vodička, R., Mantič, V.: On solvability of a boundary integral equation of the first kind for Dirichlet boundary value problems in plane elasticity. Computational Mechanics 41(6), 817-826 (2008)

29. Yan, Y., Sloan, I.H.: On integral equations of the first kind with logarithmic kernels. Journal of Integral Equations and Applications 1(4), 549-579 (1988) 